\title{
Observation of the wave-packet oscillation during the exciton self-trapping process in a quasi-one-dimensional halogen-bridged Pt complex
}

\author{
S. Tomimoto, S. Saito, and T. Suemoto \\ Institute for Solid State Physics, University of Tokyo, Kashiwa-shi, Chiba 277-8581, Japan \\ K. Sakata, J. Takeda, and S. Kurita \\ Department of Physics, Faculty of Engineering, Yokohama National University, Yokohama 240-8501, Japan
}

(Received 1 June 1999)

\begin{abstract}
Using femtosecond time-resolved luminescence spectroscopy, we have observed a wave-packet oscillation in an adiabatic potential well associated with a self-trapped exciton (STE). We have investigated the STE luminescence band of a quasi-one-dimensional halogen-bridged platinum complex $\left[\mathrm{Pt}(\mathrm{en})_{2}\right]\left[\mathrm{Pt}(\mathrm{en})_{2} \mathrm{Br}_{2}\right]\left(\mathrm{ClO}_{4}\right)_{4}$. In the time evolution of the luminescence, we have observed an oscillation with a period of about $300 \mathrm{fs}$. This result shows the formation of a wave packet and its oscillation, which is damped with the time constant of about 430 fs. The STE's have a lifetime of about $5.5 \mathrm{ps}$ at room temperature.
\end{abstract} [S0163-1829(99)06135-4]

Recent advancement in ultrashort-pulse-laser spectroscopy has made it possible to observe physical phenomena with a time resolution comparable with the typical periods of molecular vibrations or optical phonons in crystals. Many important aspects of the chemical reaction dynamics and the lattice rearrangement process in solids have been revealed by the real time observation of the motion of the oscillating atoms or ions. ${ }^{1,2}$ It is well expressed by the oscillation of a wave packet in the adiabatic potential well. Taking advantage of the broad spectral width of femtosecond pulses, we can excite several vibrational levels at a time by one optical pulse. The coherent superposition of wave functions of the excited levels results in the formation of a wave packet. The most familiar example is molecular vibrations in small molecules. In those simple systems, the dynamics of wave packets, i.e., the formation, the loss of coherence, and the relationship to the reaction dynamics, has been extensively examined, ${ }^{1}$ and some groups have made attempts to artificially control the time evolution of wave packets aiming at the control or selection of the chemical reaction path. ${ }^{3}$ In the same way, we can expect the observation of wave packets in the local lattice rearrangement process in crystals. Tokizaki et al. observed oscillatory behavior in transient absorption signals in $\mathrm{NaCl}$, which is attributed to the oscillation of a wave packet in the adiabatic potential well of the localized exciton state. ${ }^{4}$ Nisoli et al. observed an oscillation of a wave packet in $F$ center in $\mathrm{KBr}$ by their pump-and-probe experiment. ${ }^{5}$ From the analogy of molecules, we can consider the real time observation of oscillating atoms or ions in crystals to be very important for the study of the control of photoinduced structural changes of crystals.

The crystals of halogen-bridged mixed-valence platinum complexes consist of one-dimensional (1D) chains of alternating platinum and halogen ions. They are well described as the sequence $\cdots-\mathrm{Pt}^{3-\rho}-X^{-}-\mathrm{Pt}^{3+\rho}-X^{-}-\cdots$, where $\rho$ $(0 \leqslant \rho \leqslant 1)$ represents charge disproportion on Pt ions. The halogen ions $X^{-}$are located closer to $\mathrm{Pt}^{3+\rho}$ than to $\mathrm{Pt}^{3-\rho}$. This structure is understood as a charge-density wave (CDW) realized by the displacement of halogen ions from the midpoint of Pt ions ${ }^{6}$. By the optical excitation above the absorption edge, we can make a charge-transfer (CT) exciton, i.e., a pair of $\mathrm{Pt}^{3+}$ ions, on the $1 \mathrm{D}$ chain. As it is believed that there is no potential barrier in self-trapping of excitons in 1D systems, ${ }^{2,7}$ the photoexcited free excitons are expected to relax immediately to localized self-trapped states. The self-trapped exciton (STE) luminescence of the Pt complexes generally shows hot luminescence from vibrationally excited states in the high- and low-energy tails of the luminescence band. ${ }^{8,9}$ The present authors recently showed that the vibrational relaxation of hot STE's occurs with the time constant of about $500 \mathrm{fs}$ in one of the $\mathrm{Pt}$ complexes, $\left[\mathrm{Pt}(\mathrm{en})_{2}\right]\left[\mathrm{Pt}(\mathrm{en})_{2} \mathrm{Cl}_{2}\right]\left(\mathrm{ClO}_{4}\right)_{4} \quad\left(X^{-}=\mathrm{Cl}^{-}\right.$, en $=$ ethylenediamine, hereafter we abbreviate as $\mathrm{PtCl}$ ) using femtosecond time-resolved luminescence spectroscopy. ${ }^{10}$ If we excite the free excitons by the optical pulse, whose duration is shorter than the vibration period, and the excitons preserve coherence during the immediate self-trapping process, we can expect the formation of a wave packet and its oscillation, which can be observed as an oscillation in luminescence intensity.

In this paper, we present the observation of the wavepacket oscillation in the STE potential well by time-resolved luminescence measurement. We have investigated the time evolution of the STE luminescence from another compound of the $\mathrm{Pt}$ complex, $\left[\mathrm{Pt}(\mathrm{en})_{2}\right]\left[\mathrm{Pt}(\mathrm{en})_{2} \mathrm{Br}_{2}\right]\left(\mathrm{ClO}_{4}\right)_{4} \quad\left(X^{-}\right.$ $=\mathrm{Br}^{-}$, hereafter we abbreviate as $\left.\mathrm{PtBr}\right)$. We have observed an oscillation with a period of about 300 fs and its fast disappearance. From the results, we will discuss the ultrafast relaxation dynamics of the photoexcited state in the 1D system. It includes the localization of free excitons, the damped oscillation of a phonon wave packet in the adiabatic potential well, and the annihilation of STE's.

The time evolution of luminescence has been investigated at room temperature using the frequency up-conversion technique. The single crystals of $\mathrm{PtBr}$ are prepared by the procedure described in Ref. 11. The samples are excited by optical pulses from a mode-locked Ti:sapphire laser with a repetition rate of $90 \mathrm{MHz}$. The excitation light is polarized parallel 
to the chain axis of the crystal. The photon energy $1.6 \mathrm{eV}$ corresponds to the low-energy edge of the CT exciton absorption band. ${ }^{6}$ The average excitation power is $1.5 \mathrm{~mW}$. When the crystal is excited above the absorption edge, the STE luminescence band appears around $0.8 \mathrm{eV}{ }^{6}$ The luminescence from the sample is collected by off-axis paraboloidal mirrors and is mixed with variably delayed laser pulses in a nonlinear optical crystal of $\mathrm{LiIO}_{3}$ (thickness $0.5 \mathrm{~mm}$ ). The sum frequency signal is focused into a $50-\mathrm{cm}$ double monochromator and is detected by a photomultiplier tube with a photon counter. The overall time resolution of the system is about $90 \mathrm{fs}$, which is determined by the full width at half maximum of a cross-correlation trace of the scattered excitation light and the delayed laser pulses.

Compared with the $\mathrm{PtCl}$ compound, the choice of the $\mathrm{PtBr}$ compound has three experimental advantages. First, we can use fundamental frequency pulses from a Ti:sapphire laser as an excitation light source. The energy of the CT absorption band in $\mathrm{PtBr}$ is much lower than that in $\mathrm{PtCl}^{6}{ }^{6} \mathrm{We}$ do not need second-harmonic pulses whose duration is generally rather longer than that of the fundamental ones. Thus, the time resolution in the present experiment (about $90 \mathrm{fs}$ ) is much better than that of the previously reported secondharmonic excitation experiment in $\mathrm{PtCl}$ (about $180 \mathrm{fs}$ ). ${ }^{10}$ Second, as the excitation photon energy $(1.6 \mathrm{eV})$ just corresponds to the low-energy edge of the CT exciton absorption band of $\mathrm{PtBr}$, we can make free excitons with very small kinetic energy. In the experiment in $\mathrm{PtCl},{ }^{10}$ the excitation photon energy $(3.2 \mathrm{eV})$ was in the high-energy side of the CT exciton absorption band. So, the time for the intraband energy relaxation of excitons was needed. In $\mathrm{PtBr}$, however, we can expect the self-trapping of excitons with negligible delay. It might lead to the preservation of coherence during the self-trapping process of excitons. Third, as a $\mathrm{Br}$ ion is rather heavier than a $\mathrm{Cl}$ ion, we can expect a lower frequency of the STE potential well in $\mathrm{PtBr}$ than in $\mathrm{PtCl}$. As will be described in detail afterwards, it is known that the lattice relaxation in Pt complexes is primarily due to the displacement of halogen ions in the linear chain. ${ }^{7,12}$ The low frequency increases the possibility of the experimental observation of a wave packet oscillation.

Figure 1 shows the time dependence of luminescence intensity in $\mathrm{PtBr}$ measured around the peak energy $(0.8 \mathrm{eV})$ and in the high-energy side of the luminescence band. The time dependence of luminescence is well expressed by single exponential decay. All the measurements from 0.8 to $1.2 \mathrm{eV}$ have given the lifetime of $5.5 \pm 1$ ps. This lifetime is much shorter than that in $\mathrm{PtCl}$, which is about 30 ps at room temperature. ${ }^{10}$ This shows that the STE's are less stable in $\mathrm{PtBr}$ than in $\mathrm{PtCl}$. The intensity of stationary luminescence from $\mathrm{Pt}$ complexes is known to become weaker with decrease of the CT gap energy. ${ }^{13}$ Thus it is speculated that the nonradiative decay of STE's, a conversion into soliton or polaron pairs, for instance, becomes more effective as the relaxation path of the photoexcited state with decreasing CT gap energy. The luminescence lifetime obtained here is considered to reflect the rate of the nonradiative decay. In the transient absorption measurement in $\mathrm{PtBr}$, a decay component with a lifetime of 1.4 ps has been observed. ${ }^{14}$ This component has been assigned to the annihilation of STE's. However, the luminescence lifetime observed in our measurement

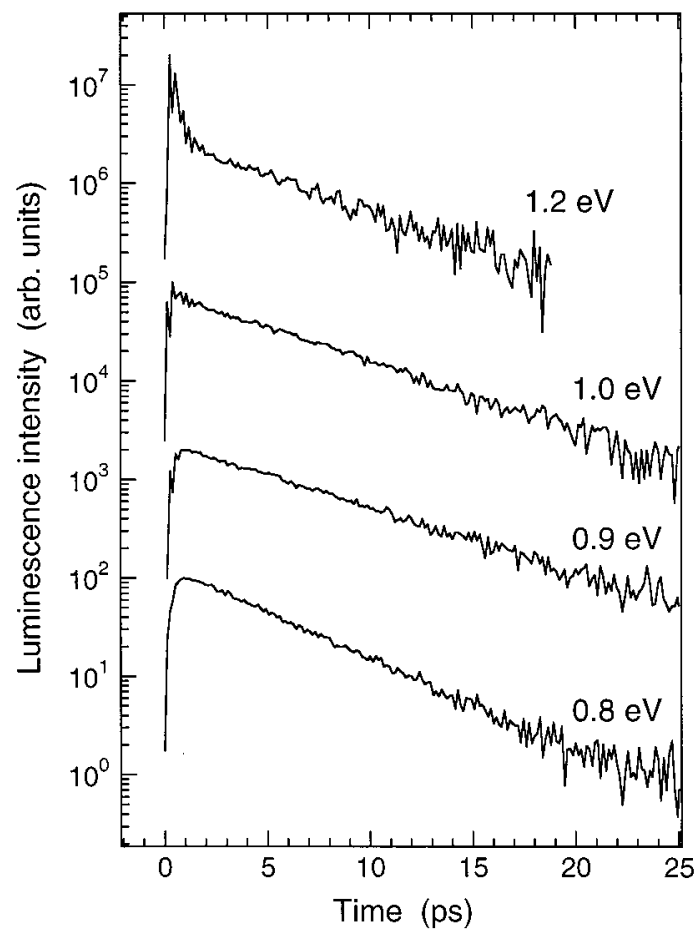

FIG. 1. Time dependence of luminescence in $\mathrm{PtBr}$ measured at room temperature. The displayed energies show the observed emission photon energy. The intensity is plotted in the logarithmic scale.

is considerably longer than $1.4 \mathrm{ps}$. The species observed in transient absorption is considered to be different from the luminescent STE's observed in our present experiment. As can be seen in the 1.2-eV curve, the additional fast decay component, which decays within 2 ps, appears in the highenergy tail of the luminescence band. The existence of the fast decay component in the tail of the luminescence band is a common feature to the Pt complexes. ${ }^{10}$ It has been attributed to hot luminescence from vibrationally excited STE's. In our experiment, a distinct oscillation is observed in that component.

The time evolution of luminescence around its onset is shown in Fig. 2 by dots. The energies displayed in the figure show the emission photon energy. The overall behavior, i.e. a relatively fast rise and decay at the high-energy tail of the luminescence band and a slow rise around the peak energy, is very similar to that in $\mathrm{PtCl}^{10}{ }^{10}$ The most striking feature of the data in Fig. 2 is the oscillation in intensity. It has a period of about 300 fs. At $1.4 \mathrm{eV}$, the first peak is followed by the very weak second peak. The second peak increases its intensity with decrease of the detection photon energy. It has almost the same intensity as the first peak at $1.1 \mathrm{eV}$. In the same way, the third peak appears and increases its intensity with decrease of the photon energy. Although the strongest peak at $1.0 \mathrm{eV}$ is the second one, the third or the fourth peak becomes the strongest one at $0.9 \mathrm{eV}$. At this energy, the oscillation can be seen for at least five periods. The oscillation, however, abruptly disappears at $0.8 \mathrm{eV}$, which is close to the STE luminescence peak energy. At this energy, only one peak is observed. At $0.7 \mathrm{eV}$, the oscillation appears again and lasts for at least three periods. Below $1.2 \mathrm{eV}$, the oscillation is followed by the slow decay of the lumines- 


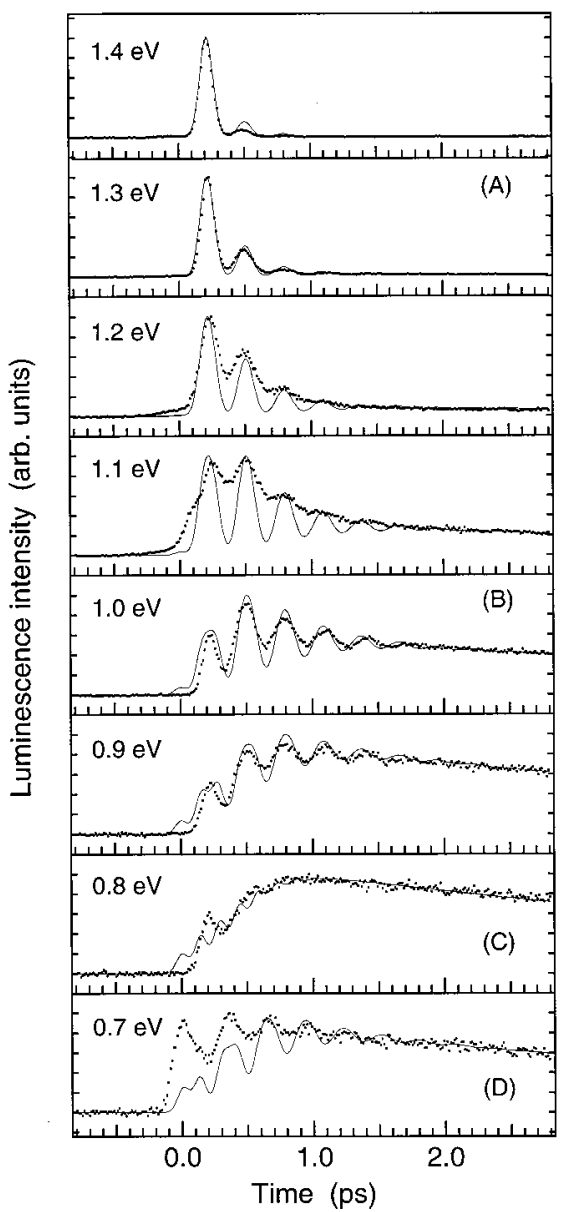

FIG. 2. Time evolution of luminescence around the onset. Dots are experimental data. Solid curves show the results of the calculation based on Eq. (4). Intensities are normalized and shown in the linear scale. The zero time delay for each photon energy has been determined by comparing the solid curves with the experimental data.

cence, which is described in the preceding paragraph. The intensity of the slow decay component relative to the first oscillation peak intensity steadily increases from 1.2 to 0.8 $\mathrm{eV}$. At $0.7 \mathrm{eV}$, however, the relative intensity becomes smaller than that at $0.8 \mathrm{eV}$.

As the excitation photon energy in this experiment (1.6 $\mathrm{eV}$ ) corresponds to the low-energy edge of the CT exciton absorption band, the excitation pulse generates free excitons, i.e., 1D Bloch state excitons, which have very small kinetic energy. Thus, the state of the system is well described by the exciton wave function with a small wave number. Such free excitons are expected to relax immediately to localized selftrapped states because there is no potential barrier between the free and self-trapped states in an ideal 1D crystal. ${ }^{2,7}$ The dynamics of excitons after the localization can be well explained using figures in Fig. 3. In Fig. 3(a), the STE on a $\mathrm{PtBr}$ chain is depicted. It is a localized pair of $\mathrm{Pt}^{3+}$ ions. The lattice relaxation in $\mathrm{PtBr}$ is primarily due to the displacement of bromine ions in the linear chain. ${ }^{7,12}$ As for the bromine ion between the two $\mathrm{Pt}^{3+}$ ions, it will move toward the central position of the two platinum ions and find a new equilibrium position. In Fig. 3(b), the adiabatic potential-energy
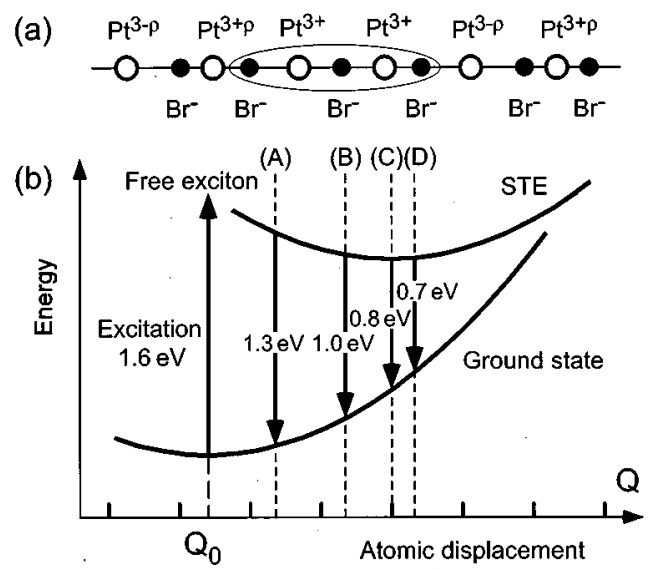

FIG. 3. (a) Schematic representation of the STE on a PtBr chain. (b) Adiabatic potential-energy curves in the configuration space.

curves are depicted. The abscissa $Q$ is the lattice distortion, which corresponds to the above-mentioned displacement of bromine ions relative to platinum ions. Here $Q_{0}$ is the configuration of the $\mathrm{CDW}$ ground state. The new equilibrium position is the bottom of the STE potential well.

The STEs just after the localization are in the excited vibrational levels in the STE state. The complicated behavior of STE luminescence within 2 ps can be well explained by the vibrational relaxation on the STE potential curve, which is expressed by the damped oscillation of a wave packet. We assume the following Gaussian-shaped wave packet at time $t$,

$$
|\psi(t, Q)|^{2}=\psi_{0} \exp \left[-\left\{\frac{Q_{c}(t)-Q}{w}\right\}^{2}\right]
$$

where $Q_{c}(t)$ and $w$ are the spatial center and extent of the wave packet. A simple damped oscillation is assumed,

$$
Q_{c}(t)=Q_{A} \exp \left(-\frac{t}{\tau_{d}}\right) \cos \left(\frac{2 \pi}{T} t+\theta\right) .
$$

The time $t=0$ represents the time of the beginning of the oscillation. The time development of the system during the transition from a Bloch state exciton to the self-localized exciton is not clear. So we leave the process to be a pending problem and assume the system to start the damped oscillation of the wave packet in the STE potential well with a certain initial position and velocity for simplicity. If the ground and excited states are expressed by two displaced harmonic potential curves of the same frequency shown in Fig. 3(b), the luminescence energy has a linear relationship with the atomic displacement $Q$ as follows,

$$
E=E_{1}-\alpha Q
$$

$E_{1}$ is the photon energy at the luminescence peak, and $\alpha$ is an electron-lattice coupling constant. If we assume that the instantaneous luminescence intensity at a given photon energy $E$ is proportional to the probability density at the corresponding transition position $Q$, we can express the luminescence intensity at time $t$ as follows, 


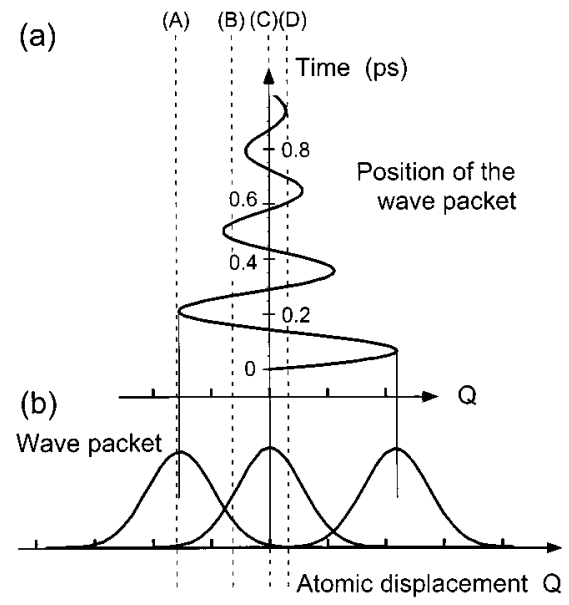

FIG. 4. (a) Time development of the central position of the wave packet, $Q_{c}(t)$, assumed in the calculation. (b) Gaussianshaped wave packet assumed in the calculation. The scale of the $Q$ axis is exactly the same as that in Fig. 3.

$$
I(t, E)=I_{0}\left|\psi\left(t, \frac{E_{1}-E}{\alpha}\right)\right|^{2} \exp \left(-\frac{t}{\tau_{\mathrm{STE}}}\right)
$$

The population of STE's is assumed to decrease with the time constant $\tau_{\mathrm{STE}}(=5.5 \mathrm{ps})$. We considered the oscillation of the wave packet which is depicted in Fig. 4(a), assuming the initial oscillation phase $\theta$ to be $\pi / 2$. The damping time constant $\tau_{d}$ and the oscillation period $T$ are assumed to be $430 \mathrm{fs}$ and $290 \mathrm{fs}$, respectively. By considering the wave packet, which has a spatial extent shown in Fig. 4(b), we have calculated the time dependence of luminescence at various photon energies. The results are shown in Fig. 2 by solid curves. They are obtained by the convolution of Eq. (4) with the experimental response function.

At 1.4 and $1.3 \mathrm{eV}$, the second peak is very weak, compared with the first peak. This shows the returning wave packet cannot get back to the position for these transition energies. The energy $1.3 \mathrm{eV}$ corresponds to the transition at position $A$ shown in Figs. 3 and 4. At 1.2 and $1.1 \mathrm{eV}$, the relative intensity of each peak is reproduced by the calculation, although the oscillation amplitude in the experimental data is smaller than that in the calculated curves. At $1.0 \mathrm{eV}$, the second peak becomes the strongest one. This means the center of the wave packet has a turning point around the position for this transition energy. Position $B$ in Figs. 3 and 4 corresponds to the transition energy of $1.0 \mathrm{eV}$. As $0.9 \mathrm{eV}$ is close to the luminescence energy from the bottom of the STE potential well, the intensity of the oscillation peak shows steady increase because of the damped oscillation of the wave packet. The rise time of the nonoscillating component of the luminescence and the lifetime of oscillation at $0.9 \mathrm{eV}$ are close to each other. At $0.8 \mathrm{eV}$, the disappearance of the oscillation is reproduced by the calculation. The disappearance of the oscillation around the peak energy can be considered as follows. As the period of the wave-packet oscillation is about $300 \mathrm{fs}$, the wave packet passes the STE potential minimum (position $C$ in Figs. 3 and 4) every $150 \mathrm{fs}$. Thus, the period of the oscillation in luminescence observed at $0.8 \mathrm{eV}$ should be $150 \mathrm{fs}$. This oscillation is hidden in our experiment because half the period ( $75 \mathrm{fs}$ ) is shorter than our experimental time resolution (90 fs). At this energy, only one peak is observed at the very early time. It is not reproduced in the calculation. At $0.7 \mathrm{eV}$ in the low-energy side of the band (position $D$ ), the oscillation due to the wave packet appears again both in experiment and calculation. However, the intensity before $500 \mathrm{fs}$ in the experiment is rather stronger than the calculated one.

Except for a few deviations, the calculated curves have reproduced some important features of the experimental data in spite of its simpleness. This shows that the vibrational relaxation is well expressed by the damped oscillation of the wave packet in the adiabatic potential well. It is known from theoretical investigations that the transient optical response of localized centers is well described by the damped oscillation of the phonon wave packet. ${ }^{15,16}$ The local vibration mode associated with a localized center is believed to be an interaction mode consisting of the linear superposition of bulk phonon modes that are extending over the whole crystal. If the frequency of the relevant bulk phonon mode has a broad spectral width, the oscillation in the interaction mode will be damped due to the dephasing among the phonon modes. The vibrational energy is dissipated to the whole crystal through the bulk modes. Thus, the dephasing of the oscillation and the energy relaxation should proceed in the same time scale. At $0.9 \mathrm{eV}$, the rise of luminescence and the decay of the oscillation have proceeded in almost the same time scale and within several vibration periods. This fact shows that the vibrational relaxation in this case is well understood by considering the STE mode to be the interaction mode, which has a broad spectral width. In $\mathrm{PtBr}$, the symmetric stretching vibration mode of bromine ions around $\mathrm{Pt}^{3+\rho}$ along the chain in the CDW ground state is known, from Raman-scattering measurement, to have the frequency of $166 \mathrm{~cm}^{-1}$ at the center of the Brillouin zone. ${ }^{17}$ The frequency of the STE mode observed in our luminescence spectroscopy (about $100 \mathrm{~cm}^{-1}$ ) is considerably lower than that. In far-infrared spectra of $\mathrm{PtBr}$, however, several vibration modes are observed around $100 \mathrm{~cm}^{-1}$. ${ }^{18}$ They might have made a significant contribution to the frequency spectrum of the STE mode.

At 1.2 and $1.1 \mathrm{eV}$, the oscillation amplitude in the experiment has been smaller than that in the calculation. In addition, the observed one oscillation peak at $0.8 \mathrm{eV}$ is not reproduced in the calculation. Although we assumed the spatial extent of the wave packet to be constant, these discrepancies show that the behavior of the wave packet is more complicated. As can be seen in Fig. 2, at $0.7 \mathrm{eV}$, the intensity of the first and second oscillation peaks relative to the slow decay component is strong compared with the calculated one. The stationary luminescence spectrum in $\mathrm{PtBr}$ is known to show asymmetry, and the intensity in the low energy side of the band is relatively weak. ${ }^{19}$ This shows that the relevant potential curves deviate from the ideal harmonic ones, which have been drawn in Fig. 3. This deviation might have caused the weak relative intensity of the slow decay component at $0.7 \mathrm{eV}$, i.e., the strong relative intensity of the first and second oscillation peaks at this energy. The anharmonicity of the potential curves should also be included in the model, if we want to construct a more realistic one. 
As shown in Fig. 4, we have assumed the generation of a wave packet at $t=0$ at the potential minimum. Before starting the wave-packet oscillation, the system should experience a transition process from a free exciton state to a localized exciton state. This is a different point of our study from the spectroscopy of molecules that has used the FranckCondon transition between the relevant potential curves for optical excitation. ${ }^{1,3}$ Before the transition process of exciton, the state of the system is well defined by the wave number $k$ of the exciton wave function. The excitons may experience the intraband energy relaxation. During the transition, the exciton wave function shrinks, and after the transition, the state of the system is described by the real-space wave function of atoms surrounding the localized exciton shown in Fig. 3. If we want to treat the relationship between the change in the spatial extent of the exciton wave function and the time development of the atomic wave function, we have to treat the electron-lattice interaction in a strict way. The transition process should have significant influence on the wave-packet dynamics especially during the first 1/4 oscillation. This process is, however, very complicated and left unsolved in this study. What we can assert here from our luminescence spectroscopy is that, in the present case of the D system, the change of the state of excitions during the self-localization process, by itself, does not lead to the destruction of the phase coherence of the established local vibrational states. We believe the detail of the transition process is a very interesting problem for the future.

In conclusion, we observed the femtosecond timeresolved luminescence from a quasi-1D $\mathrm{Pt}$ complex, $\mathrm{PtBr}$ and succeeded in the observation of the wave-packet oscillation with the period of about 300 fs. This experiment demonstrates the availability of time-resolved luminescence spectroscopy as the method of the observation of a wave packet. By observing the time dependence of luminescence over a wide emission energy range, we have been able to trace the position of the wave packet in the configuration space in real time. The experiment and calculation have shown that the vibrational relaxation in the STE is well expressed by the damped oscillation of a wave packet. It oscillates for about five periods around the STE potential minimum. The STE's nonradiatively annihilate with the lifetime of about 5.5 ps.
${ }^{1}$ E. Schreiber, Femtosecond Real-Time Spectroscopy of Small Molecules and Clusters, Springer Tracts in Modern Physics Vol. 143 (Springer-Verlag, Berlin, 1998).

${ }^{2}$ K. S. Song and R. T. Williams, Self-Trapped Excitons, Springer Series in Solid-State Sciences Vol. 105 (Springer-Verlag, Berlin, 1996).

${ }^{3}$ B. Kohler, V. V. Yakovlev, J. Che, J. L. Krause, M. Messina, K. R. Wilson, N. Schwentner, R. M. Whitnell, and Y. Yan, Phys. Rev. Lett. 74, 3360 (1995).

${ }^{4}$ T. Tokizaki, T. Makimura, H. Akiyama, A. Nakamura, K. Tanimura, and N. Itoh, Phys. Rev. Lett. 67, 2701 (1991).

${ }^{5}$ M. Nisoli, S. D. Silvestri, O. Svelto, R. Scholz, R. Fanciulli, V. Pellegrini, F. Beltram, and F. Bassani, Phys. Rev. Lett. 77, 3463 (1996).

${ }^{6}$ Y. Wada, T. Mitani, M. Yamashita, and T. Koda, J. Phys. Soc. Jpn. 54, 3143 (1985).

${ }^{7}$ M. Ueta, H. Kanzaki, K. Kobayashi, Y. Toyozawa, and E. Hanamura, Excitonic Processes in Solids, Springer Series in SolidState Sciences Vol. 60 (Springer-Verlag, Berlin, 1986).

${ }^{8}$ Y. Wada, U. Lemmer, E. O. Göbel, M. Yamashita, and K. Toriumi, Phys. Rev. B 52, 8276 (1995).
${ }^{9}$ S. Tomimoto, S. Saito, T. Suemoto, K. Sakata, J. Takeda, and S. Kurita, in Proceedings of the Third International Conference on Excitonic Processes in Condensed Matter, edited by R. T. Williams and W. M. Yen (The Electrochemical Society, Inc., Pennington, 1998), p. 338.

${ }^{10}$ S. Tomimoto, H. Nansei, S. Saito, T. Suemoto, J. Takeda, and S. Kurita, Phys. Rev. Lett. 81, 417 (1998).

${ }^{11}$ S. Kurita, S. Eguchi, S. Ohta, C. A. Arrington, and B. I. Swanson, Synth. Met. 55-57, 3341 (1993).

${ }^{12}$ A. Mishima and K. Nasu, Phys. Rev. B 39, 5758 (1989).

${ }^{13}$ H. Okamoto, T. Mitani, K. Toriumi, and M. Yamashita, Mater. Sci. Eng., B 13, L9 (1992).

${ }^{14}$ H. Ooi, M. Yamashita, and T. Kobayashi, Solid State Commun. 86, 789 (1993).

${ }^{15}$ M. Hama and M. Aihara, Phys. Rev. B 38, 1221 (1988).

${ }^{16}$ Y. Kayanuma, Phys. Rev. B 41, 3360 (1990).

${ }^{17}$ R. J. Donohoe, L. A. Worl, C. A. Arrington, A. Bulou, and B. I. Swanson, Phys. Rev. B 45, 13185 (1992).

${ }^{18}$ S. P. Love, S. C. Huckett, L. A. Worl, T. M. Frankcom, S. A. Ekberg, and B. I. Swanson, Phys. Rev. B 47, 11107 (1993).

${ }^{19}$ H. Okamoto (private communication). 